\title{
On a half-discrete Hilbert-type inequality related to hyperbolic functions
}

\section{Minghui You ${ }^{1 *}$ (i)}

"Correspondence:
youminghui@hotmail.com
${ }^{1}$ Mathematics Teaching and
Research Section, Zhejiang Institute
of Mechanical and Electrical
Engineering, Hangzhou, 310053,
China

\begin{abstract}
By the introduction of a new half-discrete kernel which is composed of several exponent functions, and using the method of weight coefficient, a Hilbert-type inequality and its equivalent forms involving multiple parameters are established. In addition, it is proved that the constant factors of the newly obtained inequalities are the best possible. Furthermore, by the use of the rational fraction expansion of the tangent function and introducing the Bernoulli numbers, some interesting and special half-discrete Hilbert-type inequalities are presented at the end of the paper.
\end{abstract}

MSC: Primary 26D15; 26D10; secondary 47B38

Keywords: Hilbert-type inequality; Half-discrete; Bernoulli number; Hyperbolic functions; Rational fraction expansion

\section{Introduction}

Let $a_{n}, \mu_{n}>0, a=\left\{a_{n}\right\}_{n=1}^{\infty}$, and $p>1$. Define

$$
l_{p, \mu}:=\left\{a:\|a\|_{p, \mu}:=\left(\sum_{k=1}^{\infty} \mu_{k} a_{k}^{p}\right)^{\frac{1}{p}}<\infty\right\} .
$$

In particular, if $\mu_{n}=1$, then we have the abbreviated notations $\|a\|_{p}:=\|a\|_{p, \mu}$ and $l_{p}:=l_{p, \mu}$. Let $p>1$ and consider measurable functions $f(x), v(x)>0$. Define

$$
L_{p, v}\left(\mathbb{R}^{+}\right):=\left\{f:\|f\|_{p, v}:=\left(\int_{\mathbb{R}^{+}} v(x) f^{p}(x) \mathrm{d} x\right)^{\frac{1}{p}}<\infty\right\} .
$$

For $v(x)=1$, we have the abbreviations as follows: $\|f\|_{p}:=\|f\|_{p, v}$ and $L_{p}\left(\mathbb{R}^{+}\right):=L_{p, v}\left(\mathbb{R}^{+}\right)$.

Consider two sequences of real numbers, $a=\left\{a_{n}\right\}_{n=1}^{\infty} \in l_{2}$ and $b=\left\{b_{n}\right\}_{n=1}^{\infty} \in l_{2}$. Then

$$
\sum_{n=1}^{\infty} \sum_{m=1}^{\infty} \frac{a_{m} b_{n}}{m+n}<\pi\|a\|_{2}\|b\|_{2},
$$

where the constant factor $\pi$ is the best possible. Inequality (1.1) was first proved by the German mathematician D. Hilbert in 1908 in his lectures on integral equations, and is

(c) The Author(s) 2021. This article is licensed under a Creative Commons Attribution 4.0 International License, which permits use, sharing, adaptation, distribution and reproduction in any medium or format, as long as you give appropriate credit to the original author(s) and the source, provide a link to the Creative Commons licence, and indicate if changes were made. The images or other third party material in this article are included in the article's Creative Commons licence, unless indicated otherwise in a credit line to the material. If material is not included in the article's Creative Commons licence and your intended use is not permitted by statutory regulation or exceeds the permitted use, you will need to obtain permission directly from the copyright holder. To view a copy of this licence, visit http://creativecommons.org/licenses/by/4.0/. 
usually known as Hilbert's double series inequality [1]. Three years later, Schur established the integral analogue of (1.1), that is[1],

$$
\int_{0}^{\infty} \int_{0}^{\infty} \frac{f(x) g(y)}{x+y} \mathrm{~d} x \mathrm{~d} y<\pi\|f\|_{2}\|g\|_{2}
$$

where $f, g \geq 0$ are two real-valued functions, and $f, g \in L_{2}\left(\mathbb{R}^{+}\right)$.

In 1925, by the introduction of a pair of conjugate parameters $p$ and $q, p>1, \frac{1}{p}+\frac{1}{q}=1$, Hardy and Riesz generalized (1.1) as follows:

$$
\sum_{n=1}^{\infty} \sum_{m=1}^{\infty} \frac{a_{m} b_{n}}{m+n}<\frac{\pi}{\sin \frac{\pi}{p}}\|a\|_{p}\|b\|_{q},
$$

where $a=\left\{a_{n}\right\}_{n=1}^{\infty} \in l_{p}$ and $b=\left\{b_{n}\right\}_{n=1}^{\infty} \in l_{q}$.

Since the 1990s, by the introduction of parameters and special functions, researchers established quite a few generalizations of (1.3) (see [2-11]).

It should be noted that there is a sharper form of (1.3) (see [1, Theorem 323]):

$$
\sum_{n=0}^{\infty} \sum_{m=0}^{\infty} \frac{a_{m} b_{n}}{m+n+1}<\frac{\pi}{\sin \frac{\pi}{p}}\|a\|_{p}\|b\|_{q}
$$

Regarding extensions of (1.4), we can refer to [12-16]. In addition, some extensions of (1.2) were also established in the past 20 years (see [10,11, 17-20]). Furthermore, by constructing new kernel functions, introducing parameters, and considering coefficient refinement, reverse form, and multi-dimensional extension, a large number of new inequalities similar to (1.1) and (1.2) were established in the past several decades (see [21-31]). These newly constructed inequalities are generally called Hilbert-type inequalities.

In addition to integral and discrete forms, Hilbert-type inequalities also appear in halfdiscrete form. The first half-discrete Hilbert-type inequality was proved by Hardy et al. [1, Theorem 351], but the constant factor was not proved to be the best possible. In 2011, Yang [32] proved that

$$
\int_{0}^{\infty} f(x) \sum_{n=1}^{\infty} \frac{a_{n}}{n+x} \mathrm{~d} x<\pi\|f\|_{2}\|a\|_{2},
$$

where the constant factor $\pi$ is the best possible. With regard to the related results of halfdiscrete Hilbert inequality, we can refer to [33-40].

The main objective of this paper is to establish a half-discrete Hilbert-type inequality with the kernel function, in particular exponential or hyperbolic function. We first present some relevant results in the literature. For example, Yang [41] proved that

$$
\int_{0}^{\infty} \int_{0}^{\infty} e^{-\frac{x}{y}} f(x) g(y) \mathrm{d} y<\|f\|_{2, v_{1}}\|g\|_{2, v_{2}},
$$

where $v_{1}(x)=x^{-1}$ and $v_{2}(y)=y^{3}$.

In 2013, Liu [42] established the following inequality involving hyperbolic secant function:

$$
\int_{0}^{\infty} \int_{0}^{\infty} \operatorname{sech}(x y) f(x) g(y) \mathrm{d} x \mathrm{~d} y<2 c_{0}\|f\|_{2, v}\|g\|_{2, v},
$$


where $\operatorname{sech}(u)=\frac{2}{e^{u}+e^{-u}}, v(x)=x^{-3}$, and $c_{0}=\sum_{k=0}^{\infty} \frac{(-1)^{k}}{(2 k+1)^{2}}=0.915965^{+}$is the Catalan constant.

Another Hilbert-type inequality with a half-discrete kernel involving hyperbolic secant function was established by Zhong [43] in 2012. It reads

$$
\int_{0}^{\infty} f(x) \sum_{n=1}^{\infty} a_{n} \operatorname{sech}\left(\frac{n}{x}\right) \mathrm{d} x<\frac{\pi}{2}\|f\|_{2, v}\|a\|_{2, \mu}
$$

where $v(x)=x^{3}, \mu_{n}=\frac{1}{n}$.

In this work, we will establish the following half-discrete Hilbert-type inequalities with the kernels involving hyperbolic tangent and cotangent functions:

$$
\begin{aligned}
& \int_{0}^{\infty} f(x) \sum_{n=1}^{\infty} a_{n}[\operatorname{coth} \sqrt[2 m]{n x}-1] \mathrm{d} x<B_{m} \pi^{2 m}\|f\|_{p, v}\|a\|_{q, \mu}, \\
& \int_{0}^{\infty} f(x) \sum_{n=1}^{\infty} a_{n}[1-\tanh \sqrt[2 m]{n x}] \mathrm{d} x<\left(1-\frac{1}{2^{2 m-1}}\right) B_{m} \pi^{2 m}\|f\|_{p, v}\|a\|_{q, \mu}
\end{aligned}
$$

where $v(x)=\frac{1}{x}, \mu_{n}=\frac{1}{n}, m \in \mathbb{N}^{+}$.

It is of interest that we also present some other half-discrete inequalities involving hyperbolic functions. More generally, we construct a kernel function with multiple parameters, which unifies the homogeneous and nonhomogeneous kernels, and then a half-discrete Hilbert-type inequality and its equivalent forms are established.

\section{Some lemmas}

Lemma 2.1 Let $\eta_{1}, \eta_{2} \in\{1,-1\}$, and $\eta_{2} \neq-1$ for $\eta_{1}=1$. Let $\gamma_{1} \in \mathbb{R}^{+}, \gamma_{2}, \gamma_{3} \in \mathbb{R}$. Suppose that $\gamma_{3} \leq \gamma_{2} \leq-\gamma_{3} \leq \gamma_{1}$ for $\eta_{2}=1$, and $\gamma_{3}<\gamma_{2} \leq-\gamma_{3} \leq \gamma_{1}$ for $\eta_{2}=-1$. Define

$$
\kappa(u):=\frac{e^{\gamma_{2} u}+\eta_{2} e^{\gamma_{3} u}}{e^{\gamma_{1} u}+\eta_{1} e^{-\gamma_{1} u}}, \quad u>0 .
$$

Then $\kappa(u)$ is decreasing on $\mathbb{R}^{+}$.

Proof It is easy to show that

$$
\begin{aligned}
\frac{\mathrm{d} \kappa}{\mathrm{d} u}= & {\left[\left(\gamma_{2}-\gamma_{1}\right) e^{\left(\gamma_{2}+\gamma_{1}\right) u}+\eta_{1}\left(\gamma_{2}+\gamma_{1}\right) e^{\left(\gamma_{2}-\gamma_{1}\right) u}\right.} \\
& \left.+\eta_{2}\left(\gamma_{3}-\gamma_{1}\right) e^{\left(\gamma_{3}+\gamma_{1}\right) u}+\eta_{1} \eta_{2}\left(\gamma_{3}+\gamma_{1}\right) e^{\left(\gamma_{3}-\gamma_{1}\right) u}\right] \\
& \times\left(e^{\gamma_{1} u}+\eta_{1} e^{-\gamma_{1} u}\right)^{-2}:=\kappa_{1}(u)\left(e^{\gamma_{1} u}+\eta_{1} e^{-\gamma_{1} u}\right)^{-2}
\end{aligned}
$$

and

$$
\begin{aligned}
\frac{\mathrm{d} \kappa_{1}}{\mathrm{~d} u}= & \left\{\left(\gamma_{2}^{2}-\gamma_{1}^{2}\right)\left[e^{\left(\gamma_{2}+\gamma_{1}\right) u}+\eta_{1} e^{\left(\gamma_{2}-\gamma_{1}\right) u}\right]\right. \\
& \left.+\eta_{2}\left(\gamma_{3}^{2}-\gamma_{1}^{2}\right)\left[e^{\left(\gamma_{3}+\gamma_{1}\right) u}+\eta_{1} e^{\left(\gamma_{3}-\gamma_{1}\right) u}\right]\right\} .
\end{aligned}
$$

For $\eta_{2}=1, \eta_{1}= \pm 1$, since $\gamma_{3} \leq \gamma_{2} \leq-\gamma_{3} \leq \gamma_{1}$, we can obtain $\gamma_{2}^{2}-\gamma_{1}^{2} \leq 0$ and $\gamma_{3}^{2}-\gamma_{1}^{2} \leq 0$. In addition, it is obvious that $e^{\left(\gamma_{2}+\gamma_{1}\right) u}+\eta_{1} e^{\left(\gamma_{2}-\gamma_{1}\right) u}>0$ and $e^{\left(\gamma_{3}+\gamma_{1}\right) u}+\eta_{1} e^{\left(\gamma_{3}-\gamma_{1}\right) u}>0$. Therefore $\frac{\mathrm{d} \kappa_{1}}{\mathrm{~d} u} \leq 0$ for $\eta_{2}=1, \eta_{1}= \pm 1$. 
For $\eta_{2}=-1, \eta_{1}=-1$, write $\kappa_{2}(t):=e^{\left(t+\gamma_{1}\right) u}-e^{\left(t-\gamma_{1}\right) u}, t \in \mathbb{R}, u>0$. Then

$$
\frac{\mathrm{d} \kappa_{2}}{\mathrm{~d} t}=u e^{\left(t+\gamma_{1}\right) u}-u e^{\left(t-\gamma_{1}\right) u}>0
$$

Therefore $\kappa_{2}(t)$ is decreasing on $\mathbb{R}$, and it follows that

$$
\begin{aligned}
\frac{\mathrm{d} \kappa_{1}}{\mathrm{~d} u} \leq & \left\{\left(\gamma_{2}^{2}-\gamma_{1}^{2}\right)\left[e^{\left(\gamma_{2}+\gamma_{1}\right) u}-e^{\left(\gamma_{2}-\gamma_{1}\right) u}\right]\right. \\
& \left.-\left(\gamma_{3}^{2}-\gamma_{1}^{2}\right)\left[e^{\left(\gamma_{2}+\gamma_{1}\right) u}-e^{\left(\gamma_{2}-\gamma_{1}\right) u}\right]\right\} \\
= & \left(\gamma_{2}^{2}-\gamma_{3}^{2}\right)\left[e^{\left(\gamma_{2}+\gamma_{1}\right) u}-e^{\left(\gamma_{2}-\gamma_{1}\right) u}\right] \leq 0 .
\end{aligned}
$$

Based on the above discussions, it follows that $\kappa_{1}(u)$ is a decreasing function on $\mathbb{R}^{+}$. Therefore, for $\eta_{1}=1, \eta_{2}=1, \kappa_{1}(u)<\kappa_{1}(0)=2\left(\gamma_{2}+\gamma_{3}\right) \leq 0$. Similarly, we can obtain that $\kappa_{1}(u)<\kappa_{1}(0)=-4 \gamma_{1}<0$ for $\eta_{1}=-1, \eta_{2}=1$, and $\kappa_{1}(u)<\kappa_{1}(0)=0$ for $\eta_{1}=-1, \eta_{2}=-1$. Applying $\kappa_{1}(u)<0$ to $(2.2)$, we get $\frac{\mathrm{d} \kappa}{\mathrm{d} u}<0$, and it follows that $\kappa(u)$ is decreasing on $\mathbb{R}^{+}$.

Lemma 2.2 Let $\eta_{1}, \eta_{2} \in\{1,-1\}$ and $\eta_{2} \neq-1$ for $\eta_{1}=1$. Let $\beta_{1} \in \mathbb{R}^{+}, \beta_{2} \in \mathbb{R} \backslash\{0\}$. Assume that $\beta \beta_{1} \leq 1, \beta \geq 1$ and $\beta \neq 1$ for $\eta_{1}=-1, \eta_{2}=1$. Let $\gamma_{1} \in \mathbb{R}^{+}, \gamma_{2}, \gamma_{3} \in \mathbb{R}$, and $\gamma_{2}, \gamma_{3} \neq \gamma_{1}$. Suppose that $\gamma_{3} \leq \gamma_{2} \leq-\gamma_{3} \leq \gamma_{1}$ for $\eta_{2}=1$, and $\gamma_{3}<\gamma_{2} \leq-\gamma_{3} \leq \gamma_{1}$ for $\eta_{2}=-1$. Define

$$
K(n, x):=\frac{e^{\gamma_{2} n^{\beta_{1}} x^{\beta_{2}}}+\eta_{2} e^{\gamma_{3} n^{\beta_{1}} x^{\beta_{2}}}}{e^{\gamma_{1} n^{\beta_{1}} x^{\beta_{2}}}+\eta_{1} e^{-\gamma_{1} n^{\beta_{1}} x^{\beta_{2}}}}, \quad n \in \mathbb{N}^{+}, x \in \mathbb{R}^{+},
$$

and

$$
C\left(\gamma_{1}, \gamma_{2}, \gamma_{3}, \eta_{1}, \eta_{2}, \beta\right)=\sum_{j=0}^{\infty}\left[\frac{\left(-\eta_{1}\right)^{j}}{\left(2 \gamma_{1} j-\gamma_{2}+\gamma_{1}\right)^{\beta}}+\frac{\eta_{2}\left(-\eta_{1}\right)^{j}}{\left(2 \gamma_{1} j-\gamma_{3}+\gamma_{1}\right)^{\beta}}\right]
$$

Then

$$
\begin{aligned}
& \omega(n):=\int_{0}^{\infty} K(n, x) x^{\beta \beta_{2}-1} \mathrm{~d} x=\frac{n^{-\beta \beta_{1}}}{\left|\beta_{2}\right|} \Gamma(\beta) C\left(\gamma_{1}, \gamma_{2}, \gamma_{3}, \eta_{1}, \eta_{2}, \beta\right), \\
& \varpi(x):=\sum_{n=1}^{\infty} K(n, x) n^{\beta \beta_{1}-1}<\frac{x^{-\beta \beta_{2}}}{\beta_{1}} \Gamma(\beta) C\left(\gamma_{1}, \gamma_{2}, \gamma_{3}, \eta_{1}, \eta_{2}, \beta\right) .
\end{aligned}
$$

Proof Setting $n^{\beta_{1}} x^{\beta_{2}}=u$, we obtain

$$
\omega(n)=\frac{n^{-\beta \beta_{1}}}{\left|\beta_{2}\right|} \int_{0}^{\infty} \kappa(u) u^{\beta-1} \mathrm{~d} u
$$

Expanding $\kappa(u)$ into a power series of $e^{u}$, we have

$$
\begin{aligned}
\kappa(u) & =\frac{e^{\left(\gamma_{2}-\gamma_{1}\right) u}+\eta_{2} e^{\left(\gamma_{3}-\gamma_{1}\right) u}}{1+\eta_{1} e^{-2 \gamma_{1} u}} \\
& =\sum_{j=0}^{\infty}\left(-\eta_{1}\right)^{j}\left[e^{\left(-2 \gamma_{1} j+\gamma_{2}-\gamma_{1}\right) u}+\eta_{2} e^{\left(-2 \gamma_{1} j+\gamma_{3}-\gamma_{1}\right) u}\right] .
\end{aligned}
$$


Therefore, by the use of Lebesgue term-by-term integration theorem, we obtain

$$
\begin{aligned}
\int_{0}^{\infty} \kappa(u) u^{\beta-1} \mathrm{~d} u= & \sum_{j=0}^{\infty}\left\{\left(-\eta_{1}\right)^{j}\left[\int_{0}^{\infty} e^{\left(-2 \gamma_{1} j+\gamma_{2}-\gamma_{1}\right) u} u^{\beta-1} \mathrm{~d} u\right]\right. \\
& \left.+\eta_{2}\left(-\eta_{1}\right)^{j}\left[\int_{0}^{\infty} e^{\left(-2 \gamma_{1} j+\gamma_{3}-\gamma_{1}\right) u} u^{\beta-1} \mathrm{~d} u\right]\right\}
\end{aligned}
$$

Setting $\left(-2 \gamma_{1} j+\gamma_{2}-\gamma_{1}\right) u=-t$, it follows that

$$
\int_{0}^{\infty} e^{\left(-2 \gamma_{1} j+\gamma_{2}-\gamma_{1}\right) u} u^{\beta-1} \mathrm{~d} u=\frac{\int_{0}^{\infty} e^{-t} t^{\beta-1} \mathrm{~d} t}{\left(2 \gamma_{1} j-\gamma_{2}+\gamma_{1}\right)^{\beta}}=\frac{\Gamma(\beta)}{\left(2 \gamma_{1} j-\gamma_{2}+\gamma_{1}\right)^{\beta}} .
$$

Similarly, we can obtain

$$
\int_{0}^{\infty} e^{\left(-2 \gamma_{1} j+\gamma_{3}-\gamma_{1}\right) u} u^{\beta-1} \mathrm{~d} u=\frac{\int_{0}^{\infty} e^{-t} t^{\beta-1} \mathrm{~d} t}{\left(2 \gamma_{1} j-\gamma_{3}+\gamma_{1}\right)^{\beta}}=\frac{\Gamma(\beta)}{\left(2 \gamma_{1} j-\gamma_{3}+\gamma_{1}\right)^{\beta}} .
$$

Applying (2.12) and (2.13) to (2.11), and using (2.6), we have

$$
\int_{0}^{\infty} \kappa(u) u^{\beta-1} \mathrm{~d} u=\Gamma(\beta) C\left(\gamma_{1}, \gamma_{2}, \gamma_{3}, \eta_{1}, \eta_{2}, \beta\right)
$$

Plugging (2.14) into (2.9), we have (2.7).

Furthermore, observing that $\beta_{1}>0$ and $\beta \beta_{1}-1<0$, by Lemma 2.1, it's easy to see that $K(n, x) n^{\beta \beta_{1}-1}$ is monotonically decreasing with respect to $n$. Therefore,

$$
\sum_{n=1}^{\infty} K(n, x) n^{\beta \beta_{1}-1}<\int_{0}^{\infty} K(u, x) u^{\beta \beta_{1}-1} \mathrm{~d} u
$$

Setting $u^{\beta_{1}} x^{\beta_{2}}=t$, and using (2.14), we obtain

$$
\begin{aligned}
\int_{0}^{\infty} K(u, x) u^{\beta \beta_{1}-1} \mathrm{~d} u & =\frac{x^{-\beta \beta_{2}}}{\beta_{1}} \int_{0}^{\infty} \kappa(t) t^{\beta-1} \mathrm{~d} t \\
& =\frac{x^{-\beta \beta_{2}}}{\beta_{1}} \Gamma(\beta) C\left(\gamma_{1}, \gamma_{2}, \gamma_{3}, \eta_{1}, \eta_{2}, \beta\right) .
\end{aligned}
$$

Combining (2.15) and (2.16), we have (2.8). The proof of Lemma 2.2 is completed.

Lemma 2.3 Let $\eta_{1}, \eta_{2} \in\{1,-1\}$ and $\eta_{2} \neq-1$ for $\eta_{1}=1$. Let $\beta_{1} \in \mathbb{R}^{+}, \beta_{2} \in \mathbb{R} \backslash\{0\}$. Assume that $\beta \beta_{1} \leq 1, \beta \geq 1$ and $\beta \neq 1$ for $\eta_{1}=-1, \eta_{2}=1$. Let $\gamma_{1} \in \mathbb{R}^{+}, \gamma_{2}, \gamma_{3} \in \mathbb{R}$, and $\gamma_{2}, \gamma_{3} \neq \gamma_{1}$. Suppose that $\gamma_{3} \leq \gamma_{2} \leq-\gamma_{3} \leq \gamma_{1}$ for $\eta_{2}=1$, and $\gamma_{3}<\gamma_{2} \leq-\gamma_{3} \leq \gamma_{1}$ for $\eta_{2}=-1$. Let $K(n, x)$ and $C\left(\gamma_{1}, \gamma_{2}, \gamma_{3}, \eta_{1}, \eta_{2}, \beta\right)$ be defined via (2.5) and (2.6), respectively. For a sufficiently small positive number $\varepsilon$, setting

$$
a(\varepsilon):=\left\{a_{n}(\varepsilon)\right\}_{n=1}^{\infty}:=\left\{n^{\frac{q\left(\beta \beta_{1}-1\right)-\beta_{1} \varepsilon}{q}}\right\}_{n=1}^{\infty}
$$

and

$$
f_{\varepsilon}(x):= \begin{cases}x^{\frac{p\left(\beta \beta_{2}-1\right)+\beta_{2} \varepsilon}{p}}, & x \in \Omega \\ 0, & x \in \mathbb{R}^{+} \backslash \Omega\end{cases}
$$


where $\Omega=\left\{x: x>0, x^{\frac{\beta_{2}}{\left|\beta_{2}\right|}}<1\right\}$. Then

$$
\begin{aligned}
\tilde{J} & :=\sum_{n=1}^{\infty} a_{n}(\varepsilon) \int_{\Omega} K(n, x) f_{\varepsilon}(x) \mathrm{d} x=\int_{\Omega} f_{\varepsilon}(x) \sum_{n=1}^{\infty} a_{n}(\varepsilon) K(n, x) \mathrm{d} x \\
& >\frac{1}{\left|\beta_{1} \beta_{2}\right|}\left[\Gamma(\beta) C\left(\gamma_{1}, \gamma_{2}, \gamma_{3}, \eta_{1}, \eta_{2}, \beta\right)+o(1)\right] .
\end{aligned}
$$

Proof By Lemma 2.1, we have

$$
\tilde{J}>\int_{\Omega} x^{\frac{p\left(\beta \beta_{2}-1\right)+\beta_{2} \varepsilon}{p}}\left[\int_{1}^{\infty} y^{\frac{q\left(\beta \beta_{1}-1\right)-\beta_{1} \varepsilon}{q}} \frac{e^{\gamma_{2} y^{\beta_{1}} x^{\beta_{2}}}+\eta_{2} e^{\gamma_{3} y^{\beta_{1}} x^{\beta_{2}}}}{e^{\gamma_{1} y^{\beta_{1}} x^{\beta_{2}}}+\eta_{1} e^{-\gamma_{1} y^{\beta_{1}} x^{\beta_{2}}}} \mathrm{~d} y\right] \mathrm{d} x .
$$

Setting $y^{\beta_{1}} x^{\beta_{2}}=u$, we get

$$
\begin{aligned}
\tilde{J}> & \frac{1}{\beta_{1}} \int_{\Omega} x^{\beta_{2} \varepsilon-1}\left[\int_{x^{\beta_{2}}}^{\infty} \kappa(u) u^{\beta-\frac{\varepsilon}{q}-1} \mathrm{~d} u\right] \mathrm{d} x \\
= & \frac{1}{\beta_{1}} \int_{\Omega} x^{\beta_{2} \varepsilon-1}\left[\int_{1}^{\infty} \kappa(u) u^{\beta-\frac{\varepsilon}{q}-1} \mathrm{~d} u\right] \mathrm{d} x \\
& +\frac{1}{\beta_{1}} \int_{\Omega} x^{\beta_{2} \varepsilon-1}\left[\int_{x^{\beta_{2}}}^{1} \kappa(u) u^{\beta-\frac{\varepsilon}{q}-1} \mathrm{~d} u\right] \mathrm{d} x \\
= & \frac{1}{\left|\beta_{1} \beta_{2}\right| \varepsilon} \int_{1}^{\infty} \kappa(u) u^{\beta-\frac{\varepsilon}{q}-1} \mathrm{~d} u+\frac{1}{\beta_{1}} \int_{\Omega} x^{\beta_{2} \varepsilon-1}\left[\int_{x^{\beta_{2}}}^{1} \kappa(u) u^{\beta-\frac{\varepsilon}{q}-1} \mathrm{~d} u\right] \mathrm{d} x .
\end{aligned}
$$

For $\beta_{2}>0$ or $\beta_{2}<0$, by Fubini's theorem, it follows that

$$
\int_{\Omega} x^{\beta_{2} \varepsilon-1}\left[\int_{x^{\beta_{2}}}^{1} \kappa(u) u^{\beta-\frac{\varepsilon}{q}-1} \mathrm{~d} u\right] \mathrm{d} x=\frac{1}{\left|\beta_{2}\right| \varepsilon} \int_{0}^{1} \kappa(u) u^{\beta+\frac{\varepsilon}{p}-1} \mathrm{~d} u .
$$

Applying (2.22) to (2.21), and using (2.14), we obtain (2.19). The proof of Lemma 2.3 is completed.

Lemma 2.4 Let $-1<z<1, \psi_{1}(u)=\tan u, \psi_{2}(u)=\sec u$, and $m \in \mathbb{N}$. Then

$$
\begin{aligned}
& \psi_{1}^{(2 m)}\left(\frac{z \pi}{2}\right)=\frac{2^{2 m+1}(2 m) !}{\pi^{2 m+1}} \sum_{j=0}^{\infty}\left[\frac{1}{(2 j+1-z)^{2 m+1}}-\frac{1}{(2 j+1+z)^{2 m+1}}\right] \\
& \psi_{1}^{(2 m+1)}\left(\frac{z \pi}{2}\right)=\frac{2^{2 m+2}(2 m+1) !}{\pi^{2 m+2}} \sum_{j=0}^{\infty}\left[\frac{1}{(2 j+1-z)^{2 m+2}}+\frac{1}{(2 j+1+z)^{2 m+2}}\right] \\
& \psi_{2}^{(2 m)}\left(\frac{z \pi}{2}\right)=\frac{2^{2 m+1}(2 m) !}{\pi^{2 m+1}} \sum_{j=0}^{\infty}\left[\frac{(-1)^{j}}{(2 j+1-z)^{2 m+1}}+\frac{(-1)^{j}}{(2 j+1+z)^{2 m+1}}\right] .
\end{aligned}
$$

Proof The rational fraction expansion of $\psi_{1}(u)=\tan u$ can be written as follows [44, 45]:

$$
\psi_{1}(u)=\tan u=2 \sum_{j=0}^{\infty}\left[\frac{1}{(2 j+1) \pi-2 u}-\frac{1}{(2 j+1) \pi+2 u}\right] .
$$


Finding the $(2 m)$ th derivative of $\psi_{1}(u)=\tan u$, we have

$$
\psi_{1}^{(2 m)}(u)=2^{2 m+1}(2 m) ! \sum_{j=0}^{\infty}\left\{\frac{1}{[(2 j+1) \pi-2 u]^{2 m+1}}-\frac{1}{[(2 j+1) \pi+2 u]^{2 m+1}}\right\} .
$$

Letting $u=\frac{z \pi}{2}$ in (2.27), we obtain (2.23). Finding the first derivative of (2.27) and letting $u=\frac{z \pi}{2}$, we arrive at (2.24). In view of

$$
2 \psi_{2}(2 u)=\psi_{1}\left(\frac{\pi}{4}+u\right)+\psi_{1}\left(\frac{\pi}{4}-u\right)
$$

and finding the $(2 m)$ th derivative of (2.28), we obtain

$$
2^{2 m+1} \psi_{2}^{(2 m)}(2 u)=\psi_{1}^{(2 m)}\left(\frac{\pi}{4}+u\right)+\psi_{1}^{(2 m)}\left(\frac{\pi}{4}-u\right) .
$$

Letting $u=\frac{z \pi}{4}$ in (2.29), and using (2.23), we have

$$
\begin{aligned}
\psi_{2}^{(2 m)}\left(\frac{z \pi}{2}\right)= & \frac{2^{2 m+1}(2 m) !}{\pi^{2 m+1}} \sum_{j=0}^{\infty}\left[\frac{1}{(4 j+1-z)^{2 m+1}}-\frac{1}{(4 j+3+z)^{2 m+1}}\right] \\
& +\frac{2^{2 m+1}(2 m) !}{\pi^{2 m+1}} \sum_{j=0}^{\infty}\left[\frac{1}{(4 j+1+z)^{2 m+1}}-\frac{1}{(4 j+3-z)^{2 m+1}}\right] \\
= & \frac{2^{2 m+1}(2 m) !}{\pi^{2 m+1}} \sum_{j=0}^{\infty}\left[\frac{(-1)^{j}}{(2 j+1-z)^{2 m+1}}+\frac{(-1)^{j}}{(2 j+1+z)^{2 m+1}}\right] .
\end{aligned}
$$

Lemma 2.4 is proved.

\section{Main results}

Theorem 3.1 Let $\eta_{1}, \eta_{2} \in\{1,-1\}$ and $\eta_{2} \neq-1$ for $\eta_{1}=1$. Let $\beta_{1} \in \mathbb{R}^{+}, \beta_{2} \in \mathbb{R} \backslash\{0\}$. Assume that $\beta \beta_{1} \leq 1, \beta \geq 1$ and $\beta \neq 1$ for $\eta_{1}=-1, \eta_{2}=1$. Let $\gamma_{1} \in \mathbb{R}^{+}, \gamma_{2}, \gamma_{3} \in \mathbb{R}$, and $\gamma_{2}, \gamma_{3} \neq \gamma_{1}$. Suppose that $\gamma_{3} \leq \gamma_{2} \leq-\gamma_{3} \leq \gamma_{1}$ for $\eta_{2}=1$, and $\gamma_{3}<\gamma_{2} \leq-\gamma_{3} \leq \gamma_{1}$ for $\eta_{2}=-1$. Let $\mu_{n}=$ $n^{q\left(1-\beta \beta_{1}\right)-1}$ and $v(x)=x^{p\left(1-\beta \beta_{2}\right)-1}$. Let $a_{n}, f(x) \geq 0$ with $a=\left\{a_{n}\right\}_{n=1}^{\infty} \in l_{q, \mu}$ and $f(x) \in L_{p, v}\left(\mathbb{R}^{+}\right)$. Consider $K(n, x)$ and $C\left(\gamma_{1}, \gamma_{2}, \gamma_{3}, \eta_{1}, \eta_{2}, \beta\right)$ defined via (2.5) and (2.6), respectively. Then the following equivalent inequalities hold true:

$$
\begin{aligned}
I_{1}: & =\sum_{n=1}^{\infty} n^{p \beta \beta_{1}-1}\left[\int_{0}^{\infty} K(n, x) f(x) \mathrm{d} x\right]^{p} \\
& <\left[\beta_{1}^{-\frac{1}{p}}\left|\beta_{2}\right|^{-\frac{1}{q}} \Gamma(\beta) C\left(\gamma_{1}, \gamma_{2}, \gamma_{3}, \eta_{1}, \eta_{2}, \beta\right)\right]^{p}\|f\|_{p, v}^{p}, \\
I_{2}: & =\int_{0}^{\infty} x^{q \beta \beta_{2}-1}\left[\sum_{n=1}^{\infty} K(n, x) a_{n}\right]^{q} \mathrm{~d} x \\
& <\left[\beta_{1}^{-\frac{1}{p}}\left|\beta_{2}\right|^{-\frac{1}{q}} \Gamma(\beta) C\left(\gamma_{1}, \gamma_{2}, \gamma_{3}, \eta_{1}, \eta_{2}, \beta\right)\right]^{q}\|a\|_{q, \mu}^{q}, \\
J:= & \sum_{n=1}^{\infty} a_{n} \int_{0}^{\infty} K(n, x) f(x) \mathrm{d} x=\int_{0}^{\infty} f(x) \sum_{n=1}^{\infty} K(n, x) a_{n} \mathrm{~d} x
\end{aligned}
$$




$$
<\beta_{1}^{-\frac{1}{p}}\left|\beta_{2}\right|^{-\frac{1}{q}} \Gamma(\beta) C\left(\gamma_{1}, \gamma_{2}, \gamma_{3}, \eta_{1}, \eta_{2}, \beta\right)\|f\|_{p, v}\|a\|_{q, \mu},
$$

where the constant factors

$$
\begin{aligned}
& {\left[\beta_{1}^{-\frac{1}{p}}\left|\beta_{2}\right|^{-\frac{1}{q}} \Gamma(\beta) C\left(\gamma_{1}, \gamma_{2}, \gamma_{3}, \eta_{1}, \eta_{2}, \beta\right)\right]^{p},} \\
& {\left[\beta_{1}^{-\frac{1}{p}}\left|\beta_{2}\right|^{-\frac{1}{q}} \Gamma(\beta) C\left(\gamma_{1}, \gamma_{2}, \gamma_{3}, \eta_{1}, \eta_{2}, \beta\right)\right]^{q},}
\end{aligned}
$$

and $\beta_{1}^{-\frac{1}{p}}\left|\beta_{2}\right|^{-\frac{1}{q}} \Gamma(\beta) C\left(\gamma_{1}, \gamma_{2}, \gamma_{3}, \eta_{1}, \eta_{2}, \beta\right)$ are the best possible.

Proof By Hölder's inequality and (2.7), we have

$$
\begin{aligned}
& {\left[\int_{0}^{\infty} K(n, x) f(x) \mathrm{d} x\right]^{p}} \\
& \quad=\left[\int_{0}^{\infty} K(n, x)\left(\frac{n^{\frac{\beta \beta_{1}-1}{p}}}{x^{\frac{\beta \beta_{2}-1}{q}}} f(x)\right)\left(\frac{x^{\frac{\beta \beta_{2}-1}{q}}}{n^{\frac{\beta \beta_{1}-1}{p}}}\right) \mathrm{d} x\right]^{p} \\
& \quad \leq \int_{0}^{\infty} K(n, x)\left[\frac{n^{\beta \beta_{1}-1}}{\left.x^{\frac{p\left(\beta \beta_{2}-1\right)}{q}} f^{p}(x)\right] \mathrm{d} x\left\{\int_{0}^{\infty} K(n, x)\left[\frac{x^{\beta \beta_{2}-1}}{n^{\frac{q\left(\beta \beta_{1}-1\right)}{p}}}\right] \mathrm{d} x\right\}^{p-1}}\right. \\
& \quad=\int_{0}^{\infty} K(n, x)\left[\frac{n^{\beta \beta_{1}-1}}{\left.x^{\frac{p\left(\beta \beta_{2}-1\right)}{q}} f^{p}(x)\right] \mathrm{d} x\left[\omega(n) n^{\frac{q\left(1-\beta \beta_{1}\right)}{p}}\right]^{p-1}}\right. \\
& \quad=\frac{n^{-p \beta \beta_{1}+\beta \beta_{1}}}{\left|\beta_{2}\right|^{p-1}\left[\Gamma(\beta) C\left(\gamma_{1}, \gamma_{2}, \gamma_{3}, \eta_{1}, \eta_{2}, \beta\right)\right]^{p-1} \int_{0}^{\infty} K(n, x) x^{\frac{p\left(1-\beta \beta_{2}\right)}{q}} f^{p}(x) \mathrm{d} x .}
\end{aligned}
$$

Therefore, by Lebesgue term-by-term integration theorem and (2.8), we obtain

$$
\begin{aligned}
I_{1} & \leq \sum_{n=1}^{\infty} \frac{n^{\beta \beta_{1}-1}}{\left|\beta_{2}\right|^{p-1}}\left[\Gamma(\beta) C\left(\gamma_{1}, \gamma_{2}, \gamma_{3}, \eta_{1}, \eta_{2}, \beta\right)\right]^{p-1} \int_{0}^{\infty} K(n, x) x^{\frac{p\left(1-\beta \beta_{2}\right)}{q}} f^{p}(x) \mathrm{d} x \\
& =\frac{1}{\left|\beta_{2}\right|^{p-1}}\left[\Gamma(\beta) C\left(\gamma_{1}, \gamma_{2}, \gamma_{3}, \eta_{1}, \eta_{2}, \beta\right)\right]^{p-1} \int_{0}^{\infty} x^{\frac{p\left(1-\beta \beta_{2}\right)}{q}} f^{p}(x) \varpi(x) \mathrm{d} x \\
& <\frac{1}{\beta_{1}\left|\beta_{2}\right|^{p-1}}\left[\Gamma(\beta) C\left(\gamma_{1}, \gamma_{2}, \gamma_{3}, \eta_{1}, \eta_{2}, \beta\right)\right]^{p} \int_{0}^{\infty} x^{p\left(1-\beta \beta_{2}\right)-1} f^{p}(x) \mathrm{d} x \\
& =\left[\beta_{1}^{-\frac{1}{p}}\left|\beta_{2}\right|^{-\frac{1}{q}} \Gamma(\beta) C\left(\gamma_{1}, \gamma_{2}, \gamma_{3}, \eta_{1}, \eta_{2}, \beta\right)\right]^{p}\|f\|_{p, v}^{p} .
\end{aligned}
$$

Hence (3.1) is proved. Similarly, by Hölder's inequality in the form of series and (2.8), we have

$$
\begin{aligned}
& {\left[\sum_{n=1}^{\infty} K(n, x) a_{n}\right]^{q}} \\
& \quad=\left[\sum_{n=1}^{\infty} K(n, x)\left(\frac{n^{\frac{\beta \beta_{1}-1}{p}}}{x^{\frac{\beta \beta_{2}-1}{q}}}\right)\left(\frac{x^{\frac{\beta \beta_{2}-1}{q}}}{n^{\frac{\beta \beta_{1}-1}{p}}} a_{n}\right)\right]^{q} \\
& \quad \leq\left[\varpi(x) x^{\frac{p\left(1-\beta \beta_{2}\right)}{q}}\right]^{q-1} \sum_{n=1}^{\infty} K(n, x) n^{\frac{q\left(1-\beta \beta_{1}\right)}{p}} a_{n}^{q} x^{\beta \beta_{2}-1}
\end{aligned}
$$




$$
<\frac{x^{-q \beta \beta_{2}+\beta \beta_{2}}}{\beta_{1}^{q-1}}\left[\Gamma(\beta) C\left(\gamma_{1}, \gamma_{2}, \gamma_{3}, \eta_{1}, \eta_{2}, \beta\right)\right]^{q-1} \sum_{n=1}^{\infty} K(n, x) n^{\frac{q\left(1-\beta \beta_{1}\right)}{p}} a_{n}^{q} .
$$

Plugging (3.6) into the left-hand side of (3.2), and using Lebesgue term-by-term integration theorem again, as well as (2.7), we obtain

$$
\begin{aligned}
I_{2} & <\frac{1}{\beta_{1}^{q-1}}\left[\Gamma(\beta) C\left(\gamma_{1}, \gamma_{2}, \gamma_{3}, \eta_{1}, \eta_{2}, \beta\right)\right]^{q-1} \int_{0}^{\infty} x^{\beta \beta_{2}-1} \sum_{n=1}^{\infty} K(n, x) n^{\frac{q\left(1-\beta \beta_{1}\right)}{p}} a_{n}^{q} \mathrm{~d} x \\
& =\frac{1}{\beta_{1}^{q-1}}\left[\Gamma(\beta) C\left(\gamma_{1}, \gamma_{2}, \gamma_{3}, \eta_{1}, \eta_{2}, \beta\right)\right]^{q-1} \sum_{n=1}^{\infty} n^{\frac{q\left(1-\beta \beta_{1}\right)}{p}} a_{n}^{q} \omega(n) \\
& =\frac{1}{\beta_{1}^{q-1}\left|\beta_{2}\right|}\left[\Gamma(\beta) C\left(\gamma_{1}, \gamma_{2}, \gamma_{3}, \eta_{1}, \eta_{2}, \beta\right)\right]^{q} \sum_{n=1}^{\infty} n^{q\left(1-\beta \beta_{1}\right)-1} a_{n}^{q} \\
& =\left[\beta_{1}^{-\frac{1}{p}}\left|\beta_{2}\right|^{-\frac{1}{q}} \Gamma(\beta) C\left(\gamma_{1}, \gamma_{2}, \gamma_{3}, \eta_{1}, \eta_{2}, \beta\right)\right]^{q}\|a\|_{q, \mu}^{q} .
\end{aligned}
$$

The proof of (3.2) is completed.

By the use of Lebesgue term-by-term integration theorem, it is obvious that there exist two forms of $J$. Using Hölder's inequality and (3.1), we have

$$
\begin{aligned}
J & =\sum_{n=1}^{\infty}\left\{\left[n^{\beta \beta_{1}-\frac{1}{p}} \int_{0}^{\infty} K(n, x) f(x) \mathrm{d} x\right]\left(a_{n} n^{-\beta \beta_{1}+\frac{1}{p}}\right)\right\} \\
& \leq I_{1}^{\frac{1}{p}}\|a\|_{q, \mu}<\beta_{1}^{-\frac{1}{p}}\left|\beta_{2}\right|^{-\frac{1}{q}} \Gamma(\beta) C\left(\gamma_{1}, \gamma_{2}, \gamma_{3}, \eta_{1}, \eta_{2}, \beta\right)\|f\|_{p, \nu}\|a\|_{q, \mu} .
\end{aligned}
$$

Therefore, we obtained (3.3) via (3.1). It will be proved that (3.3) can also be obtained via (3.2). In fact, it follows from Hölder's inequality and (3.2) that

$$
\begin{aligned}
J & =\int_{0}^{\infty}\left\{\left[x^{\beta \beta_{2}-\frac{1}{q}} \sum_{n=1}^{\infty} a_{n} K(n, x)\right]\left[x^{-\beta \beta_{2}+\frac{1}{q}} f(x)\right]\right\} \mathrm{d} x \\
& \leq I_{2}^{\frac{1}{q}}\|f\|_{p, v}<\beta_{1}^{-\frac{1}{p}}\left|\beta_{2}\right|^{-\frac{1}{q}} \Gamma(\beta) C\left(\gamma_{1}, \gamma_{2}, \gamma_{3}, \eta_{1}, \eta_{2}, \beta\right)\|f\|_{p, \nu}\|a\|_{q, \mu} .
\end{aligned}
$$

In order to prove the equivalence of (3.1), (3.2), and (3.3), we will show that both (3.1) and (3.2) hold when (3.3) is true. Let

$$
a_{n}:=n^{p \beta \beta_{1}-1}\left[\int_{0}^{\infty} K(n, x) f(x) \mathrm{d} x\right]^{p-1}
$$

It follows from (3.3) that

$$
\begin{aligned}
0 & <\left(\|a\|_{q, \mu}\right)^{p q}=\left\{\sum_{n=1}^{\infty} n^{p \beta \beta_{1}-1}\left[\int_{0}^{\infty} K(n, x) f(x) \mathrm{d} x\right]^{p}\right\}^{p} \\
& =\left[\sum_{n=1}^{\infty} a_{n} \int_{0}^{\infty} K(n, x) f(x) \mathrm{d} x\right]^{p} \\
& <\left[\beta_{1}^{-\frac{1}{p}}\left|\beta_{2}\right|^{-\frac{1}{q}} \Gamma(\beta) C\left(\gamma_{1}, \gamma_{2}, \gamma_{3}, \eta_{1}, \eta_{2}, \beta\right)\right]^{p}\|f\|_{p, v}^{p}\|a\|_{q, \mu}^{p} .
\end{aligned}
$$


Therefore,

$$
\begin{aligned}
0 & <\sum_{n=1}^{\infty} n^{p \beta \beta_{1}-1}\left[\int_{0}^{\infty} K(n, x) f(x) \mathrm{d} x\right]^{p} \\
& =\left(\|a\|_{q, \mu}\right)^{q} \\
& <\left[\beta_{1}^{-\frac{1}{p}}\left|\beta_{2}\right|^{-\frac{1}{q}} \Gamma(\beta) C\left(\gamma_{1}, \gamma_{2}, \gamma_{3}, \eta_{1}, \eta_{2}, \beta\right)\right]^{p}\|f\|_{p, v}^{p} .
\end{aligned}
$$

Hence, (3.1) is proved via (3.3). Similarly, let

$$
f(x):=x^{q \beta \beta_{2}-1}\left[\sum_{n=1}^{\infty} K(n, x) a_{n}\right]^{q-1} .
$$

By the use of (3.3), we have

$$
\begin{aligned}
0 & <\left(\|f\|_{p, v}\right)^{p q}=\left\{\int_{0}^{\infty} x^{q \beta \beta \beta_{2}-1}\left[\sum_{n=1}^{\infty} K(n, x) a_{n}\right]^{q} \mathrm{~d} x\right\}^{q} \\
& =\left[\int_{0}^{\infty} f(x) \sum_{n=1}^{\infty} K(n, x) a_{n} \mathrm{~d} x\right]^{q} \\
& <\left[\beta_{1}^{-\frac{1}{p}}\left|\beta_{2}\right|^{-\frac{1}{q}} \Gamma(\beta) C\left(\gamma_{1}, \gamma_{2}, \gamma_{3}, \eta_{1}, \eta_{2}, \beta\right)\right]^{q}\|f\|_{p, \nu}^{q}\|a\|_{q, \mu}^{q} .
\end{aligned}
$$

It follows from (3.9) that

$$
\begin{aligned}
0 & <\int_{0}^{\infty} x^{q \beta \beta_{2}-1}\left[\sum_{n=1}^{\infty} K(n, x) a_{n}\right]^{q} \mathrm{~d} x=\left(\|f\|_{p, \nu}\right)^{p} \\
& <\left[\beta_{1}^{-\frac{1}{p}}\left|\beta_{2}\right|^{-\frac{1}{q}} \Gamma(\beta) C\left(\gamma_{1}, \gamma_{2}, \gamma_{3}, \eta_{1}, \eta_{2}, \beta\right)\right]^{q}\|a\|_{q, v}^{q} .
\end{aligned}
$$

Inequality (3.2) is also proved via (3.3). According to the above discussions, (3.1), (3.2), and (3.3) are equivalent.

At last, it will be proved that the constant factors on the right-hand side of (3.1), (3.2), and (3.3) are the best possible. Assuming that the constant factor $\beta_{1}^{-\frac{1}{p}}\left|\beta_{2}\right|^{-\frac{1}{q}} \Gamma(\beta) C\left(\gamma_{1}, \gamma_{2}, \gamma_{3}, \eta_{1}\right.$, $\left.\eta_{2}, \beta\right)$ in (3.3) is not the best possible, there must be a positive number $c$ such that (3.3) still holds if $\beta_{1}^{-\frac{1}{p}}\left|\beta_{2}\right|^{-\frac{1}{q}} \Gamma(\beta) C\left(\gamma_{1}, \gamma_{2}, \gamma_{3}, \eta_{1}, \eta_{2}, \beta\right)$ is replaced with $c$. That is,

$$
\sum_{n=1}^{\infty} a_{n} \int_{0}^{\infty} K(n, x) f(x) \mathrm{d} x=\int_{0}^{\infty} f(x) \sum_{n=1}^{\infty} a_{n} K(n, x) \mathrm{d} x<c\|f\|_{p, v}\|a\|_{q, \mu} .
$$

Replacing $a_{n}$ and $f(x)$ with $a_{n}(\varepsilon)$ and $f_{\varepsilon}(x)$ defined in Lemma 2.3, respectively, and using (2.19), we have

$$
\begin{aligned}
& \frac{1}{\left|\beta_{1} \beta_{2}\right|} \Gamma(\beta) C\left(\gamma_{1}, \gamma_{2}, \gamma_{3}, \eta_{1}, \eta_{2}, \beta\right)+o(1) \\
& \quad<\varepsilon c\left\|f_{\varepsilon}\right\|_{p, v}\|a(\varepsilon)\|_{q, \mu}=\varepsilon c\left(\int_{\Omega} x^{\beta_{2} \varepsilon-1} \mathrm{~d} x\right)^{\frac{1}{p}}\left(1+\sum_{n=2}^{\infty} n^{-\beta_{1} \varepsilon-1}\right)^{\frac{1}{q}}
\end{aligned}
$$




$$
\begin{aligned}
& <\varepsilon c\left(\int_{\Omega} x^{\beta_{2} \varepsilon-1} \mathrm{~d} x\right)^{\frac{1}{p}}\left(1+\int_{1}^{\infty} x^{-\beta_{1} \varepsilon-1} \mathrm{~d} x\right)^{\frac{1}{q}} \\
& =c\left|\beta_{2}\right|^{-\frac{1}{p}}\left(\varepsilon+\beta_{1}^{-1}\right)^{\frac{1}{q}} .
\end{aligned}
$$

Let $\varepsilon \rightarrow 0^{+}$, then we obtain $\beta_{1}^{-\frac{1}{p}}\left|\beta_{2}\right|^{-\frac{1}{q}} \Gamma(\beta) C\left(\gamma_{1}, \gamma_{2}, \gamma_{3}, \eta_{1}, \eta_{2}, \beta\right) \leq c$, which contradicts the assumption, obviously. Therefore, the constant factor on the right-hand side of inequality (3.3) is the best possible. From the equivalence of (3.1), (3.2), and (3.3), the constant factors in (3.1) and (3.2) are obviously the best possible. Theorem 3.1 is proved.

\section{Corollaries}

Let $\eta_{1}=\eta_{2}=-1, \gamma_{3}=-\gamma_{2}$ and $\beta=2 m+1(m \in \mathbb{N})$ in Theorem 3.1. By the use of (2.23), we obtain the following corollary.

Corollary 4.1 Let $\beta_{1} \in \mathbb{R}^{+}, \beta_{2} \in \mathbb{R} \backslash\{0\}$, and $(2 m+1) \beta_{1} \leq 1(m \in \mathbb{N})$. Let $0<\gamma_{2}<\gamma_{1}$. Suppose that $\mu_{n}=n^{q\left[1-(2 m+1) \beta_{1}\right]-1}, v(x)=x^{p\left[1-(2 m+1) \beta_{2}\right]-1}$, and $\psi_{1}(u)=\tan u$. Let $a_{n}, f(x) \geq 0$ with $a=\left\{a_{n}\right\}_{n=1}^{\infty} \in l_{q, \mu}$ and $f(x) \in L_{p, v}\left(\mathbb{R}^{+}\right)$. Then

$$
\begin{aligned}
& \sum_{n=1}^{\infty} a_{n} \int_{0}^{\infty} \sinh \left(\gamma_{2} n^{\beta_{1}} x^{\beta_{2}}\right) \operatorname{csch}\left(\gamma_{1} n^{\beta_{1}} x^{\beta_{2}}\right) f(x) \mathrm{d} x \\
& =\int_{0}^{\infty} f(x) \sum_{n=1}^{\infty} a_{n} \sinh \left(\gamma_{2} n^{\beta_{1}} x^{\beta_{2}}\right) \operatorname{csch}\left(\gamma_{1} n^{\beta_{1}} x^{\beta_{2}}\right) \mathrm{d} x \\
& \quad<\beta_{1}^{-\frac{1}{p}}\left|\beta_{2}\right|^{-\frac{1}{q}}\left(\frac{\pi}{2 \gamma_{1}}\right)^{2 m+1} \psi_{1}^{(2 m)}\left(\frac{\gamma_{2} \pi}{2 \gamma_{1}}\right)\|f\|_{p, v}\|a\|_{q, \mu} .
\end{aligned}
$$

In particular, let $\gamma_{1}=2 \gamma, \gamma_{2}=\gamma(\gamma>0)$ in (4.1), then it follows that

$$
\begin{aligned}
& \sum_{n=1}^{\infty} a_{n} \int_{0}^{\infty} \operatorname{sech}\left(\gamma n^{\beta_{1}} x^{\beta_{2}}\right) f(x) \mathrm{d} x \\
& \quad<\beta_{1}^{-\frac{1}{p}}\left|\beta_{2}\right|^{-\frac{1}{q}} \frac{1}{2^{4 m+1}}\left(\frac{\pi}{\gamma}\right)^{2 m+1} \psi_{1}^{(2 m)}\left(\frac{\pi}{4}\right)\|f\|_{p, v}\|a\|_{q, \mu} .
\end{aligned}
$$

Setting $\gamma=1, \beta_{1}=1, \beta_{2}=-1, m=0$ in (4.2), we obtain (1.8).

In addition, let $\gamma_{1}=4 \gamma, \gamma_{2}=\gamma(\gamma>0)$ in (4.1), then we have

$$
\begin{aligned}
& \sum_{n=1}^{\infty} a_{n} \int_{0}^{\infty} \operatorname{sech}\left(\gamma n^{\beta_{1}} x^{\beta_{2}}\right) \operatorname{sech}\left(2 \gamma n^{\beta_{1}} x^{\beta_{2}}\right) f(x) \mathrm{d} x \\
& \quad<\beta_{1}^{-\frac{1}{p}}\left|\beta_{2}\right|^{-\frac{1}{q}} \frac{1}{2^{6 m+1}}\left(\frac{\pi}{\gamma}\right)^{2 m+1} \psi_{1}^{(2 m)}\left(\frac{\pi}{8}\right)\|f\|_{p, v}\|a\|_{q, \mu} .
\end{aligned}
$$

Setting $\beta_{1}=\beta_{2}=\gamma=1, m=0$ in (4.2) and (4.3), we obtain

$$
\sum_{n=1}^{\infty} a_{n} \int_{0}^{\infty} \operatorname{sech}(n x) f(x) \mathrm{d} x<\frac{\pi}{2}\|f\|_{p, v}\|a\|_{q, \mu},
$$




$$
\sum_{n=1}^{\infty} a_{n} \int_{0}^{\infty} \operatorname{sech}(n x) \operatorname{sech}(2 n x) f(x) \mathrm{d} x<\frac{(\sqrt{2}-1) \pi}{2}\|f\|_{p, v}\|a\|_{q, \mu}
$$

where $\mu_{n}=\frac{1}{n}, v(x)=\frac{1}{x}$.

Let $\eta_{1}=-1, \eta_{2}=1, \gamma_{3}=-\gamma_{2}$ and $\beta=2 m+2(m \in \mathbb{N})$ in Theorem 3.1. By the use of (2.24), we obtain the following corollary.

Corollary 4.2 Let $\beta_{1} \in \mathbb{R}^{+}, \beta_{2} \in \mathbb{R} \backslash\{0\}$, and $(2 m+2) \beta_{1} \leq 1(m \in \mathbb{N})$. Let $0 \leq \gamma_{2}<\gamma_{1}$. Suppose that $\mu_{n}=n^{q\left[1-(2 m+2) \beta_{1}\right]-1}, v(x)=x^{p\left[1-(2 m+2) \beta_{2}\right]-1}$, and $\psi_{1}(u)=\tan u$. Let $a_{n}, f(x) \geq 0$ with $a=\left\{a_{n}\right\}_{n=1}^{\infty} \in l_{q, \mu}$ and $f(x) \in L_{p, v}\left(\mathbb{R}^{+}\right)$. Then

$$
\begin{aligned}
& \sum_{n=1}^{\infty} a_{n} \int_{0}^{\infty} \cosh \left(\gamma_{2} n^{\beta_{1}} x^{\beta_{2}}\right) \operatorname{csch}\left(\gamma_{1} n^{\beta_{1}} x^{\beta_{2}}\right) f(x) \mathrm{d} x \\
& \quad=\int_{0}^{\infty} f(x) \sum_{n=1}^{\infty} \cosh \left(\gamma_{2} n^{\beta_{1}} x^{\beta_{2}}\right) \operatorname{csch}\left(\gamma_{1} n^{\beta_{1}} x^{\beta_{2}}\right) a_{n} \mathrm{~d} x \\
& \quad<\beta_{1}^{-\frac{1}{p}}\left|\beta_{2}\right|^{-\frac{1}{q}}\left(\frac{\pi}{2 \gamma_{1}}\right)^{2 m+2} \psi_{1}^{(2 m+1)}\left(\frac{\gamma_{2} \pi}{2 \gamma_{1}}\right)\|f\|_{p, v}\|a\|_{q, \mu} .
\end{aligned}
$$

Particularly, let $\gamma_{1}=\gamma>0, \gamma_{2}=0$ in (4.6), then it follows that

$$
\begin{aligned}
& \sum_{n=1}^{\infty} a_{n} \int_{0}^{\infty} \operatorname{csch}\left(\gamma n^{\beta_{1}} x^{\beta_{2}}\right) f(x) \mathrm{d} x \\
& \quad<\beta_{1}^{-\frac{1}{p}}\left|\beta_{2}\right|^{-\frac{1}{q}}\left(\frac{\pi}{2 \gamma}\right)^{2 m+2} \psi_{1}^{(2 m+1)}(0)\|f\|_{p, v}\|a\|_{q, \mu} .
\end{aligned}
$$

Additionally, letting $\gamma_{1}=2 \gamma, \gamma_{2}=\gamma(\gamma>0)$ in (4.6), we have

$$
\begin{aligned}
& \sum_{n=1}^{\infty} a_{n} \int_{0}^{\infty} \operatorname{csch}\left(\gamma n^{\beta_{1}} x^{\beta_{2}}\right) f(x) \mathrm{d} x \\
& \quad<\beta_{1}^{-\frac{1}{p}}\left|\beta_{2}\right|^{-\frac{1}{q}} \frac{1}{2^{4 m+3}}\left(\frac{\pi}{\gamma}\right)^{2 m+2} \psi_{1}^{(2 m+1)}\left(\frac{\pi}{4}\right)\|f\|_{p, v}\|a\|_{q, \mu} .
\end{aligned}
$$

Compare (4.7) with (4.8). By (2.24), it is easy to show that $\psi_{1}^{(2 m+1)}(0)=\frac{1}{2^{2 m+1}} \psi_{1}^{(2 m+1)}\left(\frac{\pi}{4}\right)$. Therefore, (4.7) and (4.8) are equivalent. Setting $\beta_{1}=\beta_{2}=\frac{1}{2}, \gamma=1, m=0$ in (4.7), we obtain

$$
\sum_{n=1}^{\infty} a_{n} \int_{0}^{\infty} \operatorname{csch}(\sqrt{n x}) f(x) \mathrm{d} x<\frac{\pi^{2}}{2}\|f\|_{p, v}\|a\|_{q, \mu}
$$

where $\mu_{n}=\frac{1}{n}, v(x)=\frac{1}{x}$.

Furthermore, letting $\gamma_{1}=4 \gamma, \gamma_{2}=\gamma(\gamma>0)$ in (4.6), we have

$$
\begin{aligned}
& \sum_{n=1}^{\infty} a_{n} \int_{0}^{\infty} \operatorname{csch}\left(\gamma n^{\beta_{1}} x^{\beta_{2}}\right) \operatorname{sech}\left(2 \gamma n^{\beta_{1}} x^{\beta_{2}}\right) f(x) \mathrm{d} x \\
& \quad<\beta_{1}^{-\frac{1}{p}}\left|\beta_{2}\right|^{-\frac{1}{q}} \frac{1}{2^{6 m+3}}\left(\frac{\pi}{\gamma}\right)^{2 m+2} \psi_{1}^{(2 m+1)}\left(\frac{\pi}{8}\right)\|f\|_{p, v}\|a\|_{q, \mu} .
\end{aligned}
$$


Let $\eta_{1}=-1, \eta_{2}=1, \gamma_{1}=\gamma, \gamma_{2}=\gamma_{3}=-\gamma$, and $\beta=2 m+2(m \in \mathbb{N})$ in Theorem 3.1. By the following equality

$$
\sum_{j=0}^{\infty} \frac{1}{(j+1)^{2 m+2}}=\frac{2^{2 m+1}}{(2 m+2) !} \pi^{2 m+2} B_{m+1},
$$

where $B_{m+1}$ is Bernoulli number, $B_{1}=\frac{1}{6}, B_{2}=\frac{1}{30}, B_{3}=\frac{1}{42}, \ldots$ Then, the following corollary holds.

Corollary 4.3 Let $\gamma, \beta_{1} \in \mathbb{R}^{+}, \beta_{2} \in \mathbb{R} \backslash\{0\}$, and $(2 m+2) \beta_{1} \leq 1(m \in \mathbb{N})$. Suppose that $\mu_{n}=$ $n^{q\left[1-(2 m+2) \beta_{1}\right]-1}, v(x)=x^{p\left[1-(2 m+2) \beta_{2}\right]-1}$, and $\psi_{2}(u)=\sec u$. Let $a_{n}, f(x) \geq 0$ with $a=\left\{a_{n}\right\}_{n=1}^{\infty} \in$ $l_{q, \mu}$ and $f(x) \in L_{p, v}\left(\mathbb{R}^{+}\right)$. Then

$$
\begin{aligned}
& \sum_{n=1}^{\infty} a_{n} \int_{0}^{\infty}\left[\operatorname{coth}\left(\gamma n^{\beta_{1}} x^{\beta_{2}}\right)-1\right] f(x) \mathrm{d} x \\
& \quad=\int_{0}^{\infty} f(x) \sum_{n=1}^{\infty}\left[\operatorname{coth}\left(\gamma n^{\beta_{1}} x^{\beta_{2}}\right)-1\right] a_{n} \mathrm{~d} x \\
& \quad<\beta_{1}^{-\frac{1}{p}}\left|\beta_{2}\right|^{-\frac{1}{q}} \frac{B_{m+1}}{2 m+2}\left(\frac{\pi}{\gamma}\right)^{2 m+2}\|f\|_{p, v}\|a\|_{q, \mu} .
\end{aligned}
$$

Setting $\beta_{1}=\beta_{2}=\frac{1}{2 m+2}, \gamma=1$, and replacing $m+1$ with $m$, we obtain (1.9).

Let $\eta_{1}=\eta_{2}=1, \gamma_{3}=-\gamma_{2}$ and $\beta=2 m+1(m \in \mathbb{N})$ in Theorem 3.1. By the use of (2.25), we obtain Corollary 4.4 .

Corollary 4.4 Let $\beta_{1} \in \mathbb{R}^{+}, \beta_{2} \in \mathbb{R} \backslash\{0\}$, and $(2 m+1) \beta_{1} \leq 1(m \in \mathbb{N})$. Let $0 \leq \gamma_{2}<\gamma_{1}$. Suppose that $\mu_{n}=n^{q\left[1-(2 m+1) \beta_{1}\right]-1}, v(x)=x^{p\left[1-(2 m+1) \beta_{2}\right]-1}$, and $\psi_{2}(u)=\sec u$. Let $a_{n}, f(x) \geq 0$ with $a=\left\{a_{n}\right\}_{n=1}^{\infty} \in l_{q, \mu}$ and $f(x) \in L_{p, v}\left(\mathbb{R}^{+}\right)$. Then

$$
\begin{aligned}
& \sum_{n=1}^{\infty} a_{n} \int_{0}^{\infty} \cosh \left(\gamma_{2} n^{\beta_{1}} x^{\beta_{2}}\right) \operatorname{sech}\left(\gamma_{1} n^{\beta_{1}} x^{\beta_{2}}\right) f(x) \mathrm{d} x \\
& \quad=\int_{0}^{\infty} f(x) \sum_{n=1}^{\infty} \cosh \left(\gamma_{2} n^{\beta_{1}} x^{\beta_{2}}\right) \operatorname{sech}\left(\gamma_{1} n^{\beta_{1}} x^{\beta_{2}}\right) a_{n} \mathrm{~d} x \\
& <\beta_{1}^{-\frac{1}{p}}\left|\beta_{2}\right|^{-\frac{1}{q}}\left(\frac{\pi}{2 \gamma_{1}}\right)^{2 m+1} \psi_{2}^{(2 m)}\left(\frac{\gamma_{2} \pi}{2 \gamma_{1}}\right)\|f\|_{p, v}\|a\|_{q, \mu} .
\end{aligned}
$$

Let $\gamma_{1}=\gamma>0, \gamma_{2}=0$ in (4.12), we have

$$
\begin{aligned}
& \sum_{n=1}^{\infty} a_{n} \int_{0}^{\infty} \operatorname{sech}\left(\gamma n^{\beta_{1}} x^{\beta_{2}}\right) f(x) \mathrm{d} x \\
& \quad<\beta_{1}^{-\frac{1}{p}}\left|\beta_{2}\right|^{-\frac{1}{q}}\left(\frac{\pi}{2 \gamma}\right)^{2 m+1} \psi_{2}^{(2 m)}(0)\|f\|_{p, v}\|a\|_{q, \mu} .
\end{aligned}
$$


Comparing (4.2) with (4.13), it can be shown that (4.2) and (4.13) are equivalent. In fact, in view of

$$
\sum_{j=0}^{\infty}\left[\frac{1}{(4 j+1)^{2 m+1}}-\frac{1}{(4 j+3)^{2 m+1}}\right]=\sum_{j=0}^{\infty} \frac{(-1)^{j}}{(2 j+1)^{2 m+1}},
$$

and using (2.23) and (2.25), we have $\psi_{1}^{(2 m)}\left(\frac{\pi}{4}\right)=2^{2 m} \psi_{2}^{(2 m)}(0)$. Therefore, (4.2) is equivalent to (4.13).

Additionally, letting $\gamma_{1}=2 \gamma, \gamma_{2}=\gamma(\gamma>0)$ in (4.12), we have

$$
\begin{aligned}
& \sum_{n=1}^{\infty} a_{n} \int_{0}^{\infty} \operatorname{csch}\left(\gamma_{1} n^{\beta_{1}} x^{\beta_{2}}\right) \tanh \left(2 \gamma_{1} n^{\beta_{1}} x^{\beta_{2}}\right) f(x) \mathrm{d} x \\
& \quad<\beta_{1}^{-\frac{1}{p}}\left|\beta_{2}\right|^{-\frac{1}{q}} \frac{1}{2^{4 m+1}}\left(\frac{\pi}{\gamma}\right)^{2 m+1} \psi_{1}^{(2 m)}\left(\frac{\pi}{4}\right)\|f\|_{p, v}\|a\|_{q, \mu} .
\end{aligned}
$$

Let $\eta_{1}=\eta_{2}=1, \gamma_{1}=\gamma, \gamma_{2}=\gamma_{3}=-\gamma$, and $\beta=2 m+2(m \in \mathbb{N})$. Due to the following equality $[44,45]$ :

$$
\sum_{j=0}^{\infty} \frac{(-1)^{j}}{(j+1)^{2 m+2}}=\frac{2^{2 m+1}-1}{(2 m+2) !} \pi^{2 m+2} B_{m+1},
$$

the following corollary holds.

Corollary 4.5 Let $\gamma, \beta_{1} \in \mathbb{R}^{+}, \beta_{2} \in \mathbb{R} \backslash\{0\}$, and $(2 m+2) \beta_{1} \leq 1(m \in \mathbb{N})$. Suppose that $\mu_{n}=$ $n^{q\left[1-(2 m+2) \beta_{1}\right]-1}, v(x)=x^{p\left[1-(2 m+2) \beta_{2}\right]-1}$, and $\psi_{2}(u)=\sec u$. Let $a_{n}, f(x) \geq 0$ with $a=\left\{a_{n}\right\}_{n=1}^{\infty} \in$ $l_{q, \mu}$ and $f(x) \in L_{p, v}\left(\mathbb{R}^{+}\right)$. Then

$$
\begin{aligned}
& \sum_{n=1}^{\infty} a_{n} \int_{0}^{\infty}\left[1-\tanh \left(\gamma n^{\beta_{1}} x^{\beta_{2}}\right)\right] f(x) \mathrm{d} x \\
& \quad=\int_{0}^{\infty} f(x) \sum_{n=1}^{\infty} a_{n}\left[1-\tanh \left(\gamma n^{\beta_{1}} x^{\beta_{2}}\right)\right] \mathrm{d} x \\
& \quad<\beta_{1}^{-\frac{1}{p}}\left|\beta_{2}\right|^{-\frac{1}{q}} \frac{2^{2 m+1}-1}{m+1}\left(\frac{\pi}{2 \gamma}\right)^{2 m+2} B_{m+1}\|f\|_{p, v}\|a\|_{q, \mu} .
\end{aligned}
$$

Setting $\beta_{1}=\beta_{2}=\frac{1}{2 m+2}, \gamma=1$, and replacing $m+1$ with $m$, we arrive at (1.10).

\section{Acknowledgements}

The author is indebted to the anonymous referees for their valuable suggestions and comments that helped improve the paper significantly.

\section{Funding}

This research was supported by the incubation foundation of Zhejiang Institute of Mechanical and Electrical Engineering (A-0271-21-206).

Availability of data and materials Not applicable. 
Authors' contributions

The author carried out the results, and read and approved the current version of the manuscript.

\section{Publisher's Note}

Springer Nature remains neutral with regard to jurisdictional claims in published maps and institutional affiliations.

Received: 25 May 2021 Accepted: 31 August 2021 Published online: 10 September 2021

\section{References}

1. Hardy, G.H., Littlewood, J.E., Pólya, G.: Inequalities. Cambridge University Press, London (1952)

2. Yang, B.C.: On an extension of Hardy-Hilbert's inequality. Chin. Ann. Math., Ser. A 23(2), $247-254$ (2002)

3. Yang, B.C., Debnath, L.: On the extended Hardy-Hilbert's inequality. J. Math. Anal. Appl. 272(1), 187-199 (2002)

4. Yang, B.C., Debnath, L.: On a new generalization of Hardy-Hilbert's inequality and its application. J. Math. Anal. Appl. 23(2), 484-497 (1999)

5. Yang, B.C.: On new extensions of Hilbert's inequality. Acta Math. Hung. 104(4), 291-299 (2004)

6. You, M.H.: On an extension of the discrete Hilbert inequality and applications. J. Wuhan Univ. Natur. Sci. Ed. 67(2), 179-184 (2021)

7. Gao, M.Z., Yang, B.C.: On the extended Hilbert's inequality. Proc. Am. Math. Soc. 126(3), 751-759 (1998)

8. Krnić, M., Pečarić, J.: Extension of Hilbert's inequality. J. Math. Anal. Appl. 324, 150-160 (2006)

9. Krnić, M., Pečarić, J., Vuković, P.: Discrete Hilbert-type inequalities with general homogeneous kernels. Rend. Circ. Mat. Palermo 60(1), 161-171 (2011)

10. Krnić, M., Pečarić, J., Perić, I., et al.: Advances in Hilbert-Type Inequalities. Element Press, Zagreb (2012)

11. Yang, B.C.: The Norm of Operator and Hilbert-Type Inequalities. Science Press, Beijing (2009)

12. Yang, B.C.: On a new extension of Hardy-Hilbert's inequality with some parameters. Acta Math. Hung. 108(4), 337-350 (2005)

13. Chen, Z., Xu, J.: New extensions of Hardy-Hilbert's inequality with multiple parameters. Acta Math. Hung. 117(4), 383-400 (2007)

14. Yang, B.C.: On a dual Hardy-Hilbert's inequality and its generalization. Anal. Math. 31(2), 151-161 (2005)

15. Kuang, J.C., Debnath, L.: On new generalizations of Hilbert's inequality and their applications. J. Math. Anal. Appl. 245(1), 248-265 (2002)

16. You, M.H.: On a new discrete Hilbert-type inequality and application. Math. Inequal. Appl. 18(4), 1575-1578 (2015)

17. Yang, B.C.: On an extension of Hilbert's integral inequality with some parameters. Aust. J. Math. Anal. Appl. 1(1), 1-8 (2004)

18. Yang, B.C.: A note on Hilbert's integral inequalities. Chin. Q. J. Math. 13(4), $83-85$ (1998)

19. Yang, B.C.: On Hilbert's integral inequality. J. Math. Anal. Appl. 220(2), 778-785 (1998)

20. Krnić, M., Pečarić, J.: General Hilbert's and Hardy's inequalities. Math. Inequal. Appl. 8(4), 29-51 (2005)

21. Rassias, M.T., Yang, B.C.: On a Hilbert-type integral inequality in the whole plane related to the extended Riemann zeta function. Complex Anal. Oper. Theory 13(4), 1765-1782 (2019)

22. Rassias, M.T., Yang, B.C.: On a Hilbert-type integral inequality related to the extended Hurwitz zeta function in the whole plane. Acta Appl. Math. 160(1), 67-80 (2019)

23. Rassias, M.T., Yang, B.C.: A Hilbert-type integral inequality in the whole plane related to the hypergeometric function and the beta function. J. Math. Anal. Appl. 428(2), 1286-1308 (2015)

24. Rassias, M.T., Yang, B.C.: On an equivalent property of a reverse Hilbert-type integral inequality related to the extended Hurwitz zeta function. J. Math. Inequal. 13(2), 315-334 (2019)

25. Rassias, M.T., Yang, B.C., Raigorodskii, A.: On a more accurate reverse Hilbert-type inequality in the whole plane. J. Math. Inequal. 14(4), 1359-1374 (2020)

26. Rassias, M.T., Yang, B.C., Raigorodskii, A.: Two kinds of the reverse Hardy-type integral inequalities with the equivalent forms related to the extended Riemann zeta function. Appl. Anal. Discrete Math. 12(2), 273-296 (2018)

27. Hong, Y., He, B., Yang, B.C.: Necessary and sufficient conditions for the validity of Hilbert-type inequalities with a class of quasi-homogeneous kernels and its applications in operator theory. J. Math. Inequal. 12(3), 777-788 (2018)

28. Hong, Y., Liao, J.Q., Yang, B.C., et al.: A class of Hilbert-type multiple integral inequalities with the kernel of generalized homogeneous function and its applications. J. Inequal. Appl. 2020, 140 (2020). https://doi.org/10.1186/s13660-020-02401-0

29. Mo, H.M., Yang, B.C.: On a new Hilbert-type integral inequality involving the upper limit functions. J. Inequal. Appl. 2020, 5 (2020). https://doi.org/10.1186/s13660-019-2280-7

30. Liu, Q:: A Hilbert-type integral inequality under configuring free power and its applications. J. Inequal. Appl. 2019), 91 (2019). https://doi.org/10.1186/s13660-019-2039-1

31. You, M.H.: On a class of Hillbert-type inequalities in the whole plane related to exponent function. J. Inequal. Appl. 2021, 33 (2021). https://doi.org/10.1186/s13660-021-02563-5

32. Yang, B.C.: A half-discrete Hilbert's inequality. J. Guangdong Univ. Educ. 31(3), 1-7 (2011)

33. Rassias, M.T., Yang, B.C.: On half-discrete Hilbert's inequality. Appl. Math. Comput. 220, 75-93 (2013)

34. Rassias, M.T., Yang, B.C., Raigorodskii, A.: On a half-discrete Hilbert-type inequality in the whole plane with the kernel of hyperbolic secant function related to the Hurwitz zeta function. In: Trigonometric Sums and Their Applications, pp. 229-259. Springer, Berlin (2020)

35. Yang, B.C., Chen, Q.: A half-discrete Hilbert-type inequality with a homogeneous kernel and an extension. J. Inequal. Appl. 2011, 124 (2011). https://doi.org/10.1186/1029-242X-2011-124

36. He, B., Yang, B.C., Chen, Q.: A new multiple half-discrete Hilbert-type inequality with parameters and a best possible constant factor. Mediterr. J. Math. 12, 1227-1244 (2015). https://doi.org/10.1007/s00009-014-0468-0

37. Yang, B.C., Wu, S.H., Wang, A.Z.: On a reverse half-discrete Hardy-Hilbert's inequality with parameters. Mathematics 7(11), 1054 (2019). https://doi.org/10.3390/math7111054

38. Yang, B.C.: A mixed Hilbert-type inequality with a best constant factor. Int. J. Pure Appl. Math. 20(3), 319-328 (2005) 
39. Yang, B.C., Debnath, L.: Half-Discrete Hilbert-Type Inequalities. World Scientific, Singapore (2014)

40. Krnić, M., Pečarić, J., Vuković, P.: A unified treatment of half-discrete Hilbert-type inequalities with a homogeneous kernel. Mediterr. J. Math. 10, 1697-1716 (2013)

41. Yang, B.C.: A new Hilbert-type integral inequality with the homogeneous kernel of degree 0. J. Zhejiang Univ. Sci. Ed. 39(4), 390-392 (2012)

42. Liu, Q., Long, S.C.: A Hilbert-type integral inequality with the kernel of hyperbolic secant function. J. Zhejiang Univ. Sci. Ed. 40(3), 255-259 (2013)

43. Zhong, J.H., Chen, Q.: A half-discrete Hilbert-type inequality with the deceasing and homogeneous kernel of degree 0. J. Zhejiang Univ. Sci. Ed. 42(1), 77-81 (2015)

44. Richard, C.F.J.: Introduction to Calculus and Analysis. Springer, New York (1989)

45. Wang, Z.X., Guo, D.R.: Introduction to Special Functions. Higher Education Press, Beijing (2012)

\section{Submit your manuscript to a SpringerOpen ${ }^{\circ}$} journal and benefit from:

- Convenient online submission

- Rigorous peer review

Open access: articles freely available online

- High visibility within the field

- Retaining the copyright to your article

Submit your next manuscript at $\boldsymbol{\Delta}$ springeropen.com 